\title{
State and Fault Estimation of Singular Delayed LPV Systems via Proportional-Integral Observer
}

\author{
Amir Hossein Hassanabadi, Masoud Shafiee and Vicenç Puig
}

\begin{abstract}
In this paper, a singular LPV system with multiple time varying delays in state is considered. The system is subject to input disturbances and actuator faults. First, the considered system is transformed to polytopic representation and then a polytopic proportional-integral unknown input observer (PI-UIO) is designed for it. This observer can estimate both the system states and the actuator faults in the system. The conditions for disturbance decoupling and also the existence and convergence of the PI-UIO are combined and then obtained in a set of LMIs in the polytope vertices. Actuator fault diagnosis is achieved via the fault estimates provided by the observer. The applicability of the proposed scheme is illustrated via a numerical example.
\end{abstract}

\section{INTRODUCTION}

The increasing demand for safety, reliability and higher efficiency in different industrial plants has turned fault diagnosis systems to be important. Model based approaches such as unknown input observers (UIOs), $H_{\infty}$ filters and parity space methods have been widely developed in recent years $[1,2]$. These methods are based on analytic redundancy which is provided by the mathematical model of the system. Faulty situation is detected when the input-output behavior of the system deviates from what is expected from the system nominal model. Unmodeled dynamics and unknown inputs can also lead to such a deviation; thus robustness of these methods is an important matter to be considered in the design procedure of fault diagnosis algorithms.

Estimation of the size of fault which is the last step in diagnosis is needed for adapting the controller with the faulty situation in order to preserve the stability and performance of the controlled system. Fault estimation has been carried out with different approaches. Descriptor approach has been used to estimate sensor faults in which the faults are considered as auxiliary states and are estimated with a suitable unknown input observer designed for the augmented system [3-5]. For estimating actuator faults, different approaches such as proportional-integral (PI) observer [6-8] and adaptive observer [9-11] have been applied. The advantage of adaptive observer compared with the PI observer is that it can estimate time varying faults in

A. H. Hassanabadi and M. Shafiee are with Department of Electrical Engineering, Amirkabir University of Technology (Tehran Polytechnic), Tehran, Iran. Email: a.hassanabadi@aut.ac.ir,mshafiee@aut.ac.ir.

V. Puig is with Automatic Control Departament, Universitat Politècnica de Catalunya ' BarcelonaTech (UPC), Barcelona, Spain. Email: vicenc.puig@upc.edu. addition to constant faults. In the current study, proportional-integral observer will be developed to estimate both the system states and actuator faults in singular delayed linear parameter varying (LPV) systems.

Singular delayed LPV systems have attracted the attention of the researchers recently [12-17]. These systems can present a general class of nonlinear systems which have both delayed dynamics and algebraic constraints between state variables in the LPV format. LPV representation makes it possible to extend various results obtained for LTI systems to nonlinear systems which have been linearized along the trajectory of parameter variations. Singular delayed LPV systems have been used to model open flow canal systems and sewer systems $[16,17]$. The stability, robust stability and filtering of continuous singular delayed LPV systems have been considered in [12-14] while the criteria for stability and stabilization of discrete counterparts have been obtained in [15]. Unknown input observer (UIO) for these systems has been designed in $[16,17]$. The designed UIO has been used to detect and isolates actuator faults. To the best of authors' knowledge, estimation of the size of actuator faults via PI observer for these systems has not been considered previously. Fault estimation is important to design and implement active fault tolerant controllers.

In this paper, singular delayed LPV systems subject to disturbances and actuator faults are considered. Proportional-integral unknown input observer (PI-UIO) is developed for these systems. This observer provides state and fault estimates while decouples the effect of unknown inputs on the estimation. The conditions for unknown input decoupling and existence of such an observer are mixed with the conditions for convergence of the estimation error and formulated in a set of LMIs in the vertices of the polytope of parameter variation domain.

This paper is organized as follows: In Section II, the problem is formulated. Section III is devoted to proportional-integral unknown input observer design. In Section IV, the main result of the paper is presented. A numerical example is used to illustrate the paper results in Section V. Section VI concludes the paper.

\section{PROBLEM FORMULATION}

In this paper, a singular delayed LPV system with the following formulation is considered: 


$$
\left\{\begin{array}{lc}
E \dot{x}(t)=\sum_{j=0}^{s} A_{j}(\theta(t)) x\left(t-\tau_{j}(t)\right)+B(\theta(t)) u(t) \\
\quad+R(\theta(t)) d(t) & F(\theta(t)) f(t) \\
y(t)=C x(t) & \\
\tau_{0}(t)=0 & j=1, \ldots, s \\
0 \leq \tau_{j}(t) \leq \tau_{m}^{j} & j=1, \ldots, s \\
\dot{\tau}_{j}(t) \leq \mu_{j} & -\tau_{m}<t<0 \\
x(t)=\phi(t) &
\end{array}\right.
$$

where $x(t) \in \mathbb{R}^{n}, \quad u(t) \in \mathbb{R}^{k_{u}}, \quad y(t) \in \mathbb{R}^{m}, \quad d(t) \in \mathbb{R}^{k_{d}}$ and $f(t) \in \mathbb{R}^{k_{f}}$ are state vector, input vector, output vector, disturbance vector and actuator fault vector respectively. In (1), $E \in \mathbb{R}^{n \times n}$ is a constant square matrix that may have rank deficiency $(\operatorname{rank}(E)=r \leq n) . A_{j}(\theta(t))$ for $j=0, \cdots, s$, $B(\theta(t)), R(\theta(t))$ and $F(\theta(t))$ are matrices with appropriate dimensions which depend on the time varying parameter vector $\theta(t) \in \mathbb{R}^{l}$ that is real time measurable. $C$ is a constant matrix with appropriate dimensions. $\tau_{j}(t)$ for $j=1, \cdots, s$ are time varying state delays and $\tau_{0}=0 . \tau_{m}^{j}$ and $\mu_{j}$ for $j=1, \cdots, s$ are the maximums of delay values and their rates of change. $\tau_{m}$ is the upper bound of all $\tau_{m}^{j} . \phi(t)$ is a continuous vector-valued initial function. The time varying parameter vector is bounded in a hyperbox as follows:

$$
\theta_{k}^{m} \leq \theta_{k}(t) \leq \theta_{k}^{M} \quad(k=1, \cdots, l) .
$$

Assumption 1. System (1) is assumed to be admissible [16].

System (1) can be presented in the following polytopic form:

$$
\left\{\begin{aligned}
E \dot{x}(t) & =\sum_{i=1}^{h} \rho_{i}(\theta(t))\left[\sum_{j=0}^{s} A_{j i} x\left(t-\tau_{j}(t)\right)\right. \\
& \left.+B_{i} u(t)+R_{i} d(t)+F_{i} f(t)\right] \\
y(t)= & C x(t)
\end{aligned}\right.
$$

where $h=2^{l}$ is the number of subsystems in the polytopic representation. $A_{j i}, B_{i}, \quad R_{i}$ and $F_{i}$ for $i=1, \cdots, h$ are matrices describing the dynamics of the subsystem in the $i^{\text {th }}$ vertex of the hyperbox. $\rho_{i}(\theta(t))$ for $i=1, \cdots, h$ are different subsystem weights which satisfy the following convex properties:

$$
\begin{aligned}
& 0 \leq \rho_{i}(\theta(t)) \leq 1 \\
& \sum_{i=1}^{h} \rho_{i}(\theta(t))=1 .
\end{aligned}
$$

\section{PI-UIO DESIGN}

In order to estimate the states and actuator faults in system (1), the following PI-UIO is considered:

$$
\left\{\begin{aligned}
\dot{z}(t) & =\sum_{i=1}^{h} \rho_{i}(\theta(t))\left[\sum _ { j = 0 } ^ { s } \left(N_{j i} z\left(t-\tau_{j}(t)\right)\right.\right. \\
& \left.\left.+L_{j i} y\left(t-\tau_{j}(t)\right)\right)+G_{i} u(t)+W_{i} \hat{f}(t)\right] \\
\hat{x}(t) & =z(t)+H_{2} y(t) \\
\hat{y}(t) & =C \hat{x}(t) \\
\dot{\hat{f}}(t) & =\sum_{i=1}^{h} \rho_{i}(\theta(t)) \Lambda_{i}(y(t)-\hat{y}(t)) \\
z(t) & =0 \quad-\tau_{m}<t<0
\end{aligned}\right.
$$

where $\hat{x}(t) \in \mathbb{R}^{n}, \hat{y}(t) \in \mathbb{R}^{m}, z(t) \in \mathbb{R}^{n}$ and $\hat{f}(t) \in \mathbb{R}^{k_{f}}$ are state estimate, output estimate, observer state and fault estimate respectively. $N_{j i}, L_{j i}, G_{i}, W_{i}, \Lambda_{i}$ and $H_{2}$ are observer matrices with appropriate dimensions that the procedure for calculating them will be provided as it is detailed in the following.

The state estimation error is:

$$
e(t)=x(t)-\hat{x}(t)
$$

that according to (3) and (6) becomes:

$e(t)=x(t)-z(t)-H_{2} C x(t)=\left(I_{n}-H_{2} C\right) x(t)-z(t)$.

If there exists a matrix $H_{1} \in \mathbb{R}^{n \times n}$ which satisfies the following condition:

$$
H_{1} E=I_{n}-H_{2} C,
$$

then (8) is converted to

$$
e(t)=H_{1} \operatorname{Ex}(t)-z(t)
$$

and the error dynamics is described by means of

$$
\dot{e}(t)=H_{1} E \dot{x}(t)-\dot{z}(t) .
$$

Substituting (3) and (6) in (11) and then doing some mathematical manipulations result the following equation:

$$
\begin{aligned}
\dot{e}(t) & =\sum_{i=1}^{h} \rho_{i}(\theta(t))\left[\sum _ { j = 0 } ^ { s } \left(N_{j i} e\left(t-\tau_{j}(t)\right)\right.\right. \\
& \left.+\left(H_{1} A_{j i}-L_{j i} C-N_{j i} H_{1} E\right) x\left(t-\tau_{j}(t)\right)\right) \\
& +\left(H_{1} B_{i}-G_{i}\right) u(t)+H_{1} R_{i} d(t) \\
& \left.+H_{1} F_{i} f(t)-W_{i} \hat{f}(t)\right] .
\end{aligned}
$$

If the following conditions are satisfied:

$$
\begin{gathered}
H_{1} E+H_{2} C=I_{n} \\
H_{1} A_{j i}-N_{j i} H_{1} E=L_{j i} C \\
G_{i}=H_{1} B_{i} \\
H_{1} R_{i}=0 \\
W_{i}=H_{1} F_{i}
\end{gathered}
$$

then the observer error dynamics can be written as 


$$
\dot{e}(t)=\sum_{i=1}^{h} \rho_{i}(\theta(t))\left[\sum_{j=0}^{s} N_{j i} e\left(t-\tau_{j}(t)\right)+W_{i} e_{f}(t)\right]
$$

where $e_{f}(t)$ is the fault estimation error defined as:

$$
e_{f}(t)=f(t)-\hat{f}(t) .
$$

For slow varying faults (with the assumption $\dot{f}(t) \simeq 0$ ), the fault estimation error dynamics is reduced to:

$$
\dot{e}_{f}(t)=-\dot{\hat{f}}(t)
$$

and using the observer formulation (6), it is transformed to:

$$
\dot{e}_{f}(t)=-\sum_{i=1}^{h} \rho_{i}(\theta(t)) \Lambda_{i} C e(t) .
$$

According to (18) and (21), the state estimation error dynamics and the fault estimation error dynamics are coupled to each other. So, to consider this coupling, (18) and (21) are augmented as follows:

$$
\dot{\bar{e}}(t)=\sum_{i=1}^{h} \rho_{i}(\theta(t)) \sum_{j=0}^{s} \bar{N}_{j i} \bar{e}\left(t-\tau_{j}(t)\right)
$$

where

$$
\begin{aligned}
& \bar{e}(t)=\left[\begin{array}{ll}
e(t)^{T} & e_{f}(t)^{T}
\end{array}\right]^{T} \\
& \bar{N}_{0 i}=\left[\begin{array}{cc}
N_{0 i} & W_{i} \\
-\Lambda_{i} C & 0
\end{array}\right] \\
& \bar{N}_{j i}=\left[\begin{array}{cc}
N_{j i} & 0 \\
0 & 0
\end{array}\right] \quad(\text { for } j=1, \cdots, s) .
\end{aligned}
$$

Now, a lemma is introduced for convergence of the PI-UIO (6) in terms of stability of (22):

Lemma 1. The LPV delayed system (22) is considered with $\tau_{m}^{j}>0$ and $\mu_{j}<1$ as maximum bounds on the size and rate of $\tau_{j}(t)$ for $j=1, \cdots, s$. This system is stable with decay rate $\alpha>0$ if there exist positive definite matrices $P$ and $Q_{j}$ for $j=1, \cdots, s$ such that the following LMIs for $i=1, \cdots, h$ are hold:

$$
\left[\begin{array}{ccccc}
\bar{\Omega}_{0}^{i} & P \bar{N}_{1 i} & P \bar{N}_{2 i} & \cdots & P \bar{N}_{s i} \\
* & \bar{Q}_{1} & 0 & \cdots & 0 \\
* & * & \bar{Q}_{2} & \cdots & 0 \\
\vdots & \vdots & \vdots & \ddots & \vdots \\
* & * & * & \cdots & \bar{Q}_{s}
\end{array}\right]<0
$$

where

$$
\begin{aligned}
& \bar{\Omega}_{0}^{i}=P \bar{N}_{0 i}+\bar{N}_{0 i}^{T} P+2 \alpha P+Q_{1}+\ldots+Q_{s}, \\
& \bar{Q}_{j}=-\left(1-\mu_{j}\right) e^{-2 \alpha \tau_{m}^{j}} Q_{j} . \quad(j=1, \cdots, s)
\end{aligned}
$$

Proof. The following Lyapunov-Krasovskii functional

$$
\begin{aligned}
V\left(t, \bar{e}_{t}\right)= & \bar{e}^{T}(t) P \bar{e}(t) \\
& +\sum_{j=1}^{s} \int_{t-\tau_{j}(t)}^{t} \bar{e}^{T}(\lambda) Q_{j} \mathrm{e}^{2 \alpha(\lambda-t)} \bar{e}(\lambda) d \lambda
\end{aligned}
$$

is considered in which $\bar{e}_{t}=: \bar{e}(t+\omega)$ where $\omega \in\left[-\tau_{m}, 0\right]$. The exponential convergence of (22) is equivalent with

$$
\Omega=\dot{V}\left(t, e_{t}\right)+2 \alpha V\left(t, e_{t}\right)<0
$$

which with some manipulations results in the sufficient condition (23) and the manipulations are omitted because of space limitation.

For calculating the PI-UIO matrices, (13) and (16) are augmented as follows:

$$
\underbrace{\left[\begin{array}{ll}
H_{1} & H_{2}
\end{array}\right]}_{\bar{H}} \underbrace{\left[\begin{array}{cccc}
E & R_{1} & \cdots & R_{h} \\
C & 0 & \cdots & 0
\end{array}\right]}_{Y}=\underbrace{\left[\begin{array}{cccc}
I_{n} & 0 & \cdots & 0
\end{array}\right]}_{\Psi}
$$

where $\bar{H} \in \mathbb{R}^{n \times(n+m)}, Y \in \mathbb{R}^{(n+m) \times\left(n+h k_{d}\right)}$ and $\Psi \in \mathbb{R}^{n \times\left(n+h k_{d}\right)}$.

Remark 1. Equation (26) is solvable if $\operatorname{rank}\left[\begin{array}{l}Y \\ \Psi\end{array}\right]=\operatorname{rank}(Y)$ [6] which is equivalent with

$$
\operatorname{rank}(Y)=n+\operatorname{rank}\left(R_{1}\right)+\ldots+\operatorname{rank}\left(R_{h}\right) .
$$

The solution of (26) is:

$$
\bar{H}=\Psi Y^{+}+K\left(I_{n+m}-Y Y^{+}\right)
$$

where $Y^{+} \in \mathbb{R}^{\left(n+h k_{d}\right) \times(n+m)}$ is the pseudo-inverse of $Y$. The term $K\left(I_{n+m}-Y Y^{+}\right)$adds additional degree of freedom to the solution which helps to design a suitable PI-UIO. Equation (28) can be partitioned as:

$$
\begin{aligned}
\bar{H} & =\left[\begin{array}{ll}
H_{1} & H_{2}
\end{array}\right]=\Psi\left[\begin{array}{ll}
Y_{1}^{+} & Y_{2}^{+}
\end{array}\right]+K\left[\begin{array}{ll}
V_{1} & V_{2}
\end{array}\right] \\
= & {\left[\begin{array}{ll}
\Psi Y_{1}^{+}+K V_{1} & \Psi Y_{2}^{+}+K V_{2}
\end{array}\right] } \\
H_{10} & H_{20}
\end{aligned}
$$

in which $Y_{1}^{+}=Y^{+} T_{1}, Y_{2}^{+}=Y^{+} T_{2}, V_{1}=V T_{1}$ and $V_{2}=V T_{2}$ are calculated based on $T_{1}=\left[\begin{array}{ll}I_{n} & 0_{n \times m}\end{array}\right]^{T}, \quad T_{2}=\left[\begin{array}{ll}0_{m \times n} & I_{m}\end{array}\right]^{T}$ and $V=I_{n+m}-Y Y^{+}$. Now, a set of new variables are defined for $j=0, \cdots, s$ and $i=1, \cdots, h$ :

$$
K_{j i}=L_{j i}-N_{j i} H_{2} .
$$

With these new variables, (14) is transformed to:

$$
N_{j i}=H_{1} A_{j i}-K_{j i} C
$$

and considering (29), it is written as:

$$
N_{j i}=H_{10} A_{j i}+K V_{1} A_{j i}-K_{j i} C .
$$




\section{MAIN RESULT}

Based on the material provided so far, the main result of the paper is introduced.

Theorem 1. If there exist positive definite matrices $P$ and $Q_{j}$ for $j=1, \cdots, s$ and matrices $M$ and $M_{j i}$ for $j=0, \cdots, s$ and $i=1, \cdots, h$ such that the following LMIs are satisfied for $i=1, \cdots, h$ :

$$
\left[\begin{array}{ccccc}
\Omega_{0}^{i} & \Omega_{1}^{i} & \Omega_{2}^{i} & \cdots & \Omega_{s}^{i} \\
* & \bar{Q}_{1} & 0 & \cdots & 0 \\
* & * & \bar{Q}_{2} & \cdots & 0 \\
\vdots & \vdots & \vdots & \ddots & \vdots \\
* & * & * & \cdots & \bar{Q}_{s}
\end{array}\right]<0
$$

where

$$
\begin{aligned}
\Omega_{0}^{i} & =\operatorname{sym}\left\{P\left[\begin{array}{cc}
H_{10} A_{0 i} & H_{10} F_{i} \\
0 & 0
\end{array}\right]+M\left[\begin{array}{ll}
V_{1} A_{0 i} & V_{1} F_{i}
\end{array}\right]\right. \\
& \left.-M_{0 i}\left[\begin{array}{ll}
C & 0
\end{array}\right]\right\}+2 \alpha P+Q_{1}+\ldots+Q_{s}
\end{aligned}
$$

and for $j=1, \cdots, s$ :

$$
\begin{aligned}
\Omega_{j}^{i} & =P\left[\begin{array}{cc}
H_{10} A_{j i} & 0 \\
0 & 0
\end{array}\right]+M\left[\begin{array}{ll}
V_{1} A_{j i} & 0
\end{array}\right] \\
& -M_{j i}\left[\begin{array}{ll}
C & 0
\end{array}\right] \\
\bar{Q}_{j} & =-\left(1-\mu_{j}\right) e^{-2 \alpha \tau_{m}^{j}} Q_{j}
\end{aligned}
$$

and $\operatorname{Sym}\{Y\}=Y+Y^{T}$ is used to simplify the notation, then the PI-UIO (6) with exponential decay rate $\alpha>0$ for system (3) exists. The matrices $K, K_{j i}$ and $\Lambda_{i}$ are calculated with:

$$
\begin{gathered}
K=\left(P\left[\begin{array}{c}
I_{n} \\
0_{k_{f} \times n}
\end{array}\right]\right)^{+} M \\
K_{0 i}=\left[\begin{array}{ll}
I_{n} & 0_{n \times k_{f}}
\end{array}\right]\left(P^{-1} M_{0 i}\right) \\
\Lambda_{i}=\left[\begin{array}{ll}
0_{k_{f} \times n} & I_{k_{f}}
\end{array}\right]\left(P^{-1} M_{0 i}\right) \\
K_{j i}=\left(P\left[\begin{array}{c}
I_{n} \\
0_{k_{f} \times n}
\end{array}\right]\right)^{+} M_{j i} \quad(j=1, \cdots, s)
\end{gathered}
$$

and then the matrices $N_{j i}, H_{2}, G_{i}, W_{i}$ and $L_{j i}$ are calculated based on (32), (29), (15), (17) and (30) respectively.

Proof. By considering (17) and (32), the matrices of augmented system (22) are formulated as:

$$
\begin{aligned}
\bar{N}_{0 i} & =\left[\begin{array}{cc}
H_{10} A_{0 i} & H_{10} F_{i} \\
0 & 0
\end{array}\right]+\left[\begin{array}{c}
K \\
0
\end{array}\right]\left[\begin{array}{ll}
V_{1} A_{0 i} & V_{1} F_{i}
\end{array}\right] \\
& -\left[\begin{array}{c}
K_{0 i} \\
\Lambda_{i}
\end{array}\right]\left[\begin{array}{ll}
C & 0
\end{array}\right]
\end{aligned}
$$

and for $j=1, \cdots, s$ :

$$
\bar{N}_{j i}=\left[\begin{array}{cc}
H_{10} A_{j i} & 0 \\
0 & 0
\end{array}\right]+\left[\begin{array}{c}
K \\
0
\end{array}\right]\left[\begin{array}{ll}
V_{1} A_{j i} & 0
\end{array}\right]-\left[\begin{array}{c}
K_{i j} \\
0
\end{array}\right]\left[\begin{array}{ll}
C & 0
\end{array}\right] .
$$

Applying Lemma 1 on system (22) and substituting (38) and (39) in LMIs (23), a set of nonlinear matrix inequalities are obtained due to multiplicative terms of some variables. The following variables are introduced to resolve the nonlinearities:

$$
\begin{gathered}
M=P\left[\begin{array}{c}
K \\
0
\end{array}\right], \\
M_{0 i}=P\left[\begin{array}{c}
K_{0 i} \\
\Lambda_{i}
\end{array}\right], \\
M_{j i}=P\left[\begin{array}{c}
K_{i j} \\
0
\end{array}\right] \quad j=1, \cdots, s .
\end{gathered}
$$

By using these change of variables, (33) is obtained which assures the PI-UIO convergence. When (33) is solved with suitable solvers, the PI-UIO matrices can be calculated. By considering $P \in \mathbb{R}^{\left(n+k_{f}\right) \times\left(n+k_{f}\right)}$ as $P=\left[\begin{array}{ll}P_{1} & P_{2}\end{array}\right], \quad$ (40) is transformed to:

$$
M=P_{1} K=\left(P\left[\begin{array}{c}
I_{n} \\
0_{k_{f} \times n}
\end{array}\right]\right) K .
$$

So, $K$ is calculated as (34). $K_{j i}$ for $j=1, \cdots, s$ is calculated in a similar manner based on (37). According to (41):

$$
\left[\begin{array}{c}
K_{0 i} \\
\Lambda_{i}
\end{array}\right]=P^{-1} M_{0 i}
$$

and based on this, $K_{0 i}$ and $\Lambda_{i}$ are calculated from (35)-(36) respectively. Other PI-UIO matrices are calculated based on the equations stated in the theorem and the mathematical manipulation is described in Section III.

\section{EXAMPLE}

In this section, an example is presented to illustrate the applicability of the proposed method. The singular delayed LPV system (1) is considered with the following numerical values: 
$A_{0}(\theta(t))=\left[\begin{array}{cccc}-1+0.2 \theta_{1}(\mathrm{t}) & -2 & 0 & 1.2+0.5 \theta_{2}(\mathrm{t}) \\ 0.5 & -3.5 & -1.3-0.3 \theta_{1}(\mathrm{t}) & 2 \\ -0.7 & -1 & -3.7 & 2.6 \\ -0.4 & -1.2 & 0.3-0.7 \theta_{2}(\mathrm{t}) & -0.5\end{array}\right]$

$A_{1}(\theta(t))=\left[\begin{array}{cccc}0 & 1+0.2 \theta_{1}(t) & 0 & -0.5 \\ -1.2 & 0 & -1+0.4 \theta_{2}(t) & 0.8 \\ 0 & 1-0.1 \theta_{1}(t) & 0 & 0 \\ -0.7 & 0 & 0.3 & 0\end{array}\right]$,

$E=\left[\begin{array}{llll}1 & 0 & 0 & 0 \\ 0 & 1 & 0 & 0 \\ 0 & 0 & 1 & 0 \\ 0 & 0 & 0 & 0\end{array}\right], C=\left[\begin{array}{cccc}-1 & 1 & 0 & 2 \\ 4 & -2 & 3 & 1 \\ 1 & 0 & -1 & 5 \\ -2 & 1 & 0 & 1\end{array}\right], R=\left[\begin{array}{c}0.7 \\ 1 \\ -0.5 \\ 0\end{array}\right]$,

$B(\theta(t))=\left[\begin{array}{cc}3.2+0.75 \theta_{1}(t) & 0 \\ 0 & 0 \\ 0 & -2.5+0.5 \theta_{2}(t) \\ 0 & 0\end{array}\right], F=\left[\begin{array}{ll}1 & 0 \\ 0 & 0 \\ 0 & 1 \\ 0 & 0\end{array}\right]$.

The value of state delay is $\tau(t)=0.6+0.4 \sin (t)$. The range of the two parameters are $\theta_{1}(t) \in[-1,1]$ and $\theta_{2}(t) \in[-1.5,1.5]$. This system is transformed to polytopic representation with the method described in [16]. The set of LMIs (33) is solved with SeDumi solver [18] via YALMIP toolbox [19]. The decay rate is set to $\alpha=0.5$. The PI-UIO matrices are calculated based on the equations described in Theorem 1. Although there are four subsystems, due to space limitation just the gain matrices for the first subsystem of PI-UIO are presented here:

$$
\begin{aligned}
& N_{01}=\left|\begin{array}{rrrr}
-2.3277 & 2.3970 & 2.7648 & -0.9801 \\
-2.3970 & -2.3277 & -1.2520 & 0.5008 \\
-2.7648 & 1.2520 & -2.3277 & -0.6132 \\
0.9801 & -0.5008 & 0.6132 & -2.3277
\end{array}\right|, \\
& N_{11}=10^{-9} \times\left[\begin{array}{rrrr}
0.1692 & -0.0680 & -0.2367 & -0.0628 \\
0.0209 & -0.0252 & 0.1965 & 0.1013 \\
0.0770 & 0.0006 & -0.1489 & 0.0111 \\
-0.0433 & -0.0042 & 0.0705 & -0.0023
\end{array} \mid\right. \text {, } \\
& L_{01}=\left[\begin{array}{rrrr}
-9.0591 & -0.4777 & 3.1530 & 5.2092 \\
-4.8309 & 0.3975 & 1.9015 & 4.4386 \\
-0.0368 & 0.0264 & 0.0362 & 0.0462 \\
0.8272 & -0.0230 & -0.3052 & -0.6283
\end{array} \mid\right. \text {, } \\
& L_{11}=\left[\begin{array}{rrrr}
1.4044 & -0.2454 & -0.2413 & -1.1280 \\
2.3614 & -0.4745 & -0.3585 & -1.9096 \\
-0.0472 & -0.0154 & 0.0261 & 0.0330 \\
-0.3058 & 0.0539 & 0.0522 & 0.2457
\end{array}\right\rfloor, \\
& \Lambda_{1}=\left[\begin{array}{rrrr}
-63.1155 & -2.3982 & 23.4940 & 38.2407 \\
28.2239 & -0.5484 & -6.6251 & -24.7359
\end{array}\right] \text {. }
\end{aligned}
$$

$$
G_{1}=\left|\begin{array}{rr}
0.0908 & -2.1787 \\
-0.0666 & -4.2032 \\
-0.0866 & -0.1324 \\
-0.0181 & 0.4785
\end{array}\right|
$$

The values of $H_{2}$ and $W$ are:

$$
\begin{gathered}
H_{2}=\left[\begin{array}{rrrr}
0.2174 & -0.1811 & 0.1270 & -0.8889 \\
0.6432 & -0.4136 & 0.0526 & -1.1360 \\
0.1170 & 0.2625 & -0.1719 & 0.3628 \\
0.0163 & 0.0917 & 0.1280 & 0.2357
\end{array}\right], \\
W=\left\lfloor\begin{array}{rr}
0.0371 & 0.6704 \\
-0.0272 & 1.2933 \\
-0.0353 & 0.0407 \\
-0.0074 & -0.1472
\end{array}\right\rfloor .
\end{gathered}
$$

The system under consideration with the designed PI-UIO has been simulated in a $300 \mathrm{sec}$ time interval. In the simulation, the input signals are $u_{1}(t)=1+\sin (t)$ and $u_{2}(t)=\cos (0.2 t)$. The variation of the two parameters are $\theta_{1}(t)=\sin (0.3 t) \quad$ and $\quad \theta_{2}(t)=1.5 \cos (0.8 t)$. The disturbance is a zero-mean noise with standard deviation equal to 0.5 . Abrupt and incipient faults on the two actuators are considered as follows:

$$
\begin{array}{r}
f_{1}(t)=\left\{\begin{array}{lr}
1 & 30 \leq t<80 \mathrm{~s} \\
(t-100) / 50 & 100 \leq t<150 \mathrm{~s} \\
0 & \text { otherwise }
\end{array}\right. \\
f_{2}(t)=\left\{\begin{array}{lr}
1 & 170 \leq t<220 \mathrm{~s} \\
(t-240) / 50 & 240 \leq t<290 \mathrm{~s} \\
0 & \text { otherwise }
\end{array}\right.
\end{array}
$$

The state estimation errors and fault estimates are depicted in Figures 1 and 2 respectively.

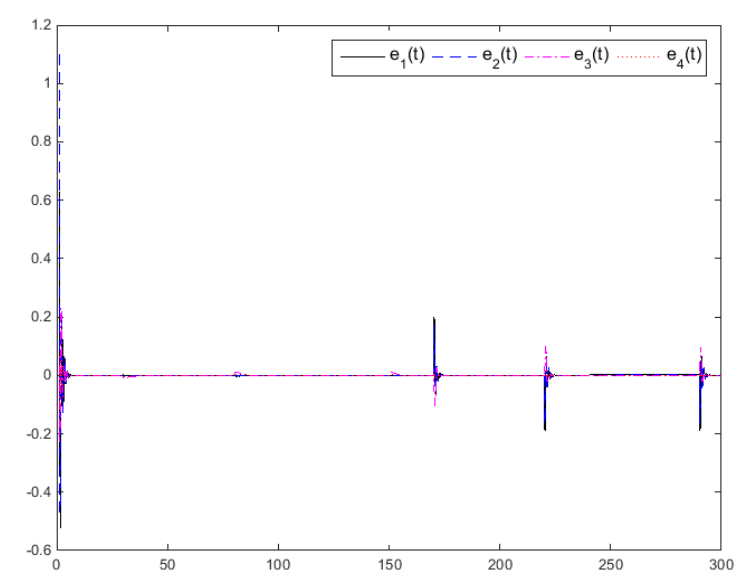

Fig 1. State estimation error

As it can be observed from Figure 1, the errors of state estimation converged to zero except the times that sudden faults happen in the system. In such times because the 
derivative of fault is very large, the assumption of slow varying faults $(\dot{f}(t) \simeq 0)$ is greatly violated so the state estimates are deviated from zero. This phenomenon can also be observed in Figure 2.
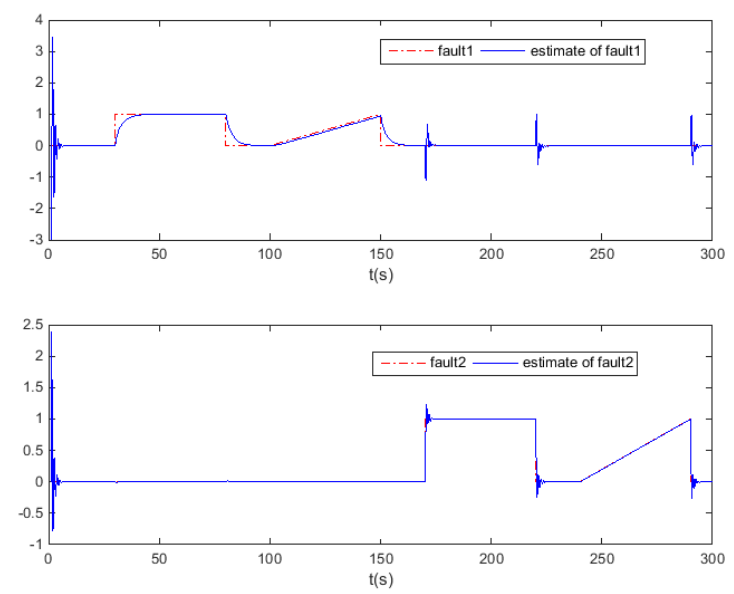

Fig 2. Fault estimates

When there is sudden change in faults, the fault estimate is deviated from its real value. But when the faults have constant values or changes with a limited slope (incipient faults), the fault estimates have good precision and the state estimation error converges to zero. Relaxing the assumption of slow varying faults is part of the future research which may be possible by extending the adaptive observer scheme proposed in [10] for singular LPV systems to the case with state delays. The approach of fault estimation proposed in this paper can be used as a direct alternative to fault diagnosis. In this approach, the faults are detected and isolated based on their estimated values. So, residual generation and residual evaluation are not needed which reduces the computational burden of the fault diagnosis unit.

\section{CONCLUSION}

In this paper, a polytopic PI-UIO was designed for singular delayed LPV systems subject to disturbances and actuator faults. The proposed observer can estimate system states and actuator faults while decoupling the disturbance effect on the estimation. The fault estimation approach is a suitable alternative for fault detection and isolation which has lower computational burden. The assumption of slow varying faults was needed to formulate the convergence of the observer. State and fault estimates are acceptable in the situations with limited-slope faults. Relaxing the assumption on fault derivative is part of future research which may be carried out with an adaptive observer scheme.

\section{REFERENCES}

S. X. Ding, Model-based fault diagnosis techniques: design schemes, algorithms, and tools: Springer Science \& Business Media, 2008

$$
\text { [1] }
$$

J. Chen and R. J. Patton, Robust model-based fault diagnosis for dynamic systems vol. 3: Springer Science \& Business Media, 2012.

Q. Jia, H. Li, Y. Zhang, and X. Chen, "Robust observer-based sensor fault reconstruction for discrete-time systems via a descriptor system approach," International Journal of Control, Automation and Systems, vol. 13, pp. 274-283, 2015.

F.-R. López-Estrada, J.-C. Ponsart, C.-M. Astorga-Zaragoza, J.L. Camas-Anzueto, and D. Theilliol, "Robust Sensor Fault Estimation for Descriptor-LPV Systems with Unmeasurable Gain Scheduling Functions: Application to an Anaerobic Bioreactor," International Journal of Applied Mathematics and Computer Science, vol. 25, pp. 233-244, 2015.

F.-R. Lopez-Estrada, J.-C. Ponsart, C. Astorga-Zaragoza, and D. Theilliol, "Fault estimation observer design for descriptor-LPV systems with unmeasurable gain scheduling functions," in Conference on Control and Fault-Tolerant Systems (SysTol), 2013 2013, pp. 269-274.

D. Koenig, "Unknown input proportional multiple-integral observer design for linear descriptor systems: application to state and fault estimation," IEEE Transactions on Automatic Control, vol. 50, pp. 212-217, 2005.

Z. Gao and S. X. Ding, "Fault estimation and fault-tolerant control for descriptor systems via proportional, multiple-integral and derivative observer design," IET Control Theory \& Applications, vol. 1, pp. 1208-1218, 2007.

H. Hamdi, M. Rodrigues, C. Mechmeche, D. Theilliol, and N. B. Braiek, "Fault detection and isolation in linear parameter-varying descriptor systems via proportional integral observer," International journal of adaptive control and signal processing, vol. 26, pp. 224-240, 2012.

K. Zhang, B. Jiang, and V. Cocquempot, "Adaptive observerbased fast fault estimation," International Journal of Control Automation and Systems, vol. 6, p. 320, 2008.

M. Rodrigues, H. Hamdi, D. Theilliol, C. Mechmeche, and N. B. Braiek, "Fault diagnosis based on adaptive polytopic observer for LPV descriptor systems," in 8th IFAC Symposium on Fault Detection, Supervision and Safety of Technical Processes (SAFEPROCESS), Mexico City, Mexico, 2012.

J. Zhang, A. K. Swain, and S. K. Nguang, "Robust Hळ adaptive descriptor observer design for fault estimation of uncertain nonlinear systems," Journal of the Franklin Institute, vol. 351, pp. 5162-5181, 11// 2014.

L. Fanbiao, Z. Xian, and W. Hui, "Stability analysis of singular lpv systems with time-varying delays: A projection approach," in 29th Chinese Control Conference (CCC), 2010 2010, pp. 944949.

F. Li and X. Zhang, "A delay-dependent bounded real lemma for singular LPV systems with time-variant delay," International Journal of Robust and Nonlinear Control, vol. 22, pp. 559-574, 2012.

F. Li and X. Zhang, "Delay-range-dependent robust $\mathrm{H} \infty$ filtering for singular LPV systems with time variant delay," International Journal of Innovative Computing, Information and Control, vol. 9, pp. 339-353, 2013.

X. Zhang and H. Zhu, "Robust Stability and Stabilization Criteria for Discrete Singular Time-Delay LPV Systems," Asian Journal of Control, vol. 14, pp. 1084-1094, 2012.

A. H. Hassanabadi, M. Shafiee, and V. Puig, "UIO design for singular delayed LPV systems with application to actuator fault detection and isolation," International Journal of Systems Science, vol. 47, pp. 107-121, 2016/01/02 2015.

A. H. Hassanabadi, M. Shafiee, and V. Puig, "Robust fault detection of singular LPV systems with multiple time-varing delays," Int. J. Appl. Math. Comput. Sci vol. 26, pp. 45-61, 2016. J. F. Sturm, "Using SeDuMi 1.02, a MATLAB toolbox for optimization over symmetric cones," Optimization methods and software, vol. 11, pp. 625-653, 1999.

J. Löfberg, "YALMIP: A toolbox for modeling and optimization in MATLAB.," in IEEE International Symposium on Computer Aided Control Systems Design, Taipei, Taiwan, 2004, pp. 284289. 